\title{
Evolution and genetic diversity of atypical porcine pestivirus (APPV) from piglets with congenital tremor in Guangxi province, Southern China
}

\section{Kaichuang Shi ( $\nabla$ shikaichuang@126.com )}

Guangxi Center for Animal Disease Control and Prevention https://orcid.org/0000-0002-1023-9635 Shou-yu Xie

Guangxi Center for Animal Disease Control and Prevention

Jing Zhao

Guangxi University

Hui-xin Liu

Guangxi Univereity

Yan-wen Yin

Guangxi Center for Animal Disease Control and Prevention

Zhenhai Chen

Yangzhou University

Hong-bin Si

Guangxi University

Su-jie Qu

GUangxi Center for Animal Disease Control and Prevention

Wen-jun Lu

GUangxi Center for Animal Disease Control and Prevention

Shu-ping Feng

Guangxi Center for Animal Disease Control and Prevention

\section{Research article}

Keywords: Atypical porcine pestivirus, Genomic characterization, Genetic diversity, Evolution

Posted Date: March 18th, 2020

DOI: https://doi.org/10.21203/rs.3.rs-17589/v1

License: (c) (1) This work is licensed under a Creative Commons Attribution 4.0 International License.

Read Full License 
Version of Record: A version of this preprint was published at Veterinary Medicine and Science on December 13th, 2020. See the published version at https://doi.org/10.1002/vms3.407. 


\section{Abstract}

Background: Atypical porcine pestivirus (APPV) was identified and associated with congenital tremor (CT) type A-II in new born piglets and has been reported in many countries around the world since 2015. In China, the first APPV infection in swine herds was reported in Guangdong province in 2016. To investigate the genetic characteristics of APPV from Guangxi province seated in Southern China, the fulllength sequences of APPV strains were amplified and analyzed.

Results: Tissue samples from neonatal piglets with CT from Guangxi province were detected by reverse transcription-polymerase chain reaction (RT-PCR). APPV positive samples were amplified, cloned and sequenced, and the complete genomic sequences of five APPV strains were obtained. Sequence analysis revealed that all six APPV strains from Guangxi province, including five strains from this study and one from other researchers, shared $83.3 \%-97.5 \%$ nucleotide identity of complete genome and $91.7 \%-99.1 \%$ amino acid identity of open reading frame (ORF) with one another, and shared $77.7 \%-97.7 \%$ nucleotide identity of complete genome and $90.6 \%-99.3 \%$ amino acid identity of ORF with other reference strains available in Genbank. Phylogenetic analysis indicated that the APPV strains from Guangxi province belonged to four different subgroups in the phylogenetic tree based on the complete genomic sequences, and similar topology was observed in the phylogenetic trees based on N pro, E rns and E2 gene sequences, respectively. No sign of recombination was observed for strains from Guangxi province by using Recombination Detection Program 4 (RDP4) and Simplot analysis. Evolution analysis performed on the complete genome of 58 APPV strains available in Genbank showed that APPV strains from America, Europe and Asia during 2006-2019 evolved at a mean rate of 1.37×10 -4 substitutions/site/year, and the most recent common ancestor ( tMRCA) of them was estimated as 1700.5 years ago.

Conclusions: The findings of this study indicated that there existed a high degree of genetic diversity of APPV from Guangxi province, Southern China, which provided important information on the epidemiological features and evolutionary relationships of APPV.

\section{Background}

One novel atypical porcine pestivirus (APPV) was first discovered and identified by next-generation sequencing (NGS) from clinical samples in the United States in 2015 [1]. Since then, the presence of APPV in new born piglets with congenital tremor (CT) has been reported in many countries worldwide [2, 3], such as USA [4], the Netherlands [5], Germany [6], Austria [7], Spain [8], Brazil [9], Hungary [10], England [11], Canada [12], Sweden [13], China [14], Korea [15], Switzerland [16], Italy [17] and so on, showing that APPV has wide geographical distribution around the world. Two independent studies have been reported that congenital tremor (CT) was reproduced following experimental inoculation with serum or tissuehomogenate-pools containing APPV, demonstrating that APPV could be a very likely causative agent of CT in piglets [4, 5]. APPV genome was identified in different tissues of the CT affected piglets from 1997 to 2016 by quantitative reverse transcription-polymerase chain reaction (qRT-PCR), suggesting that APPV has been circulating in Spain at least since 1997 [6]. APPV was identified in lymph nodes from Swedish 
pigs suffering from postweaning multisystemic wasting syndrome (PMWS) by viral metagenomic analysis, showing that APPV has been circulating in Swedish pig farms since 2007 [13]. Recently, one study indicated that APPV was detected in serum samples as far back as 1986, showing that APPV had already been circulating in the indigenous pig population in Switzerland for many decades [16]. However, the existence of APPV in serum from apparently healthy adult pigs revealed that it might prevalent for a long time without any clinical manifestations $[5,7]$. In China, the first APPV infection in swine herds was reported in Guangdong province in 2016 [14], and subsequently, the genomic presences of APPV were detected in pigs from Guangxi, Guizhou, Jiangxi, Yunnan, Anhui and other provinces in China $[18,19,20$, $21,22,23,24]$.

APPV, together with classical swine fever virus (CSFV), bovine viral diarrhea virus 1 (BVDV-1), BVDV-2 and border disease virus (BDV), belongs to the genus Pestivirus of family Flaviviridae [25]. APPV infected piglets are characterized by generalized body shaking with variable degrees of hypomyelination in brain and spinal cord. APPV has been discovered in both domestic pigs and wild boar populations [17, 26, 27], suggesting APPV has wide range of hosts. High APPV loads were detected by qRT-PCR in semen, serum and different tissue samples of clinically healthy domestic adult pigs and commercial boar studs [7, 27], indicating there existed persistent infection and this virus could possibly be transmitted by artificial insemination. Besides horizontal transmission through oronasal pathway, APPV could also be vertically transmitted by transplacental infection $[4,5]$. One retrospective study showed that $41.8 \%(51 / 122)$ sampled pig farms and 16.3\% (182/1115) porcine serum samples could be detected antibodies against APPV in Germany [28]. The infection rate of piglets born from infected sows could reach $16 \%-100 \%$, the mortality rate of piglets from infected herds could reach $30 \%$, and the survival rate of weanling piglets could drop 10\% per sow, showing that APPV could cause huge economic losses to pig industry [7, 29]. Therefore, as a newly discovered virus, APPV has attracted great attention all over the world.

APPV is a newly virus, but not a new virus. So far, many researchers focused on molecular epidemiological analysis of APPV epidemic strains and found that there existed high genetic variation among different strains $[7,11,13,14,18,20,21,22]$, with up to $21 \%$ genetic distance among the viruses [30]. However, strains available for biological, origin, and evolution analyses are still scarce so far, and it is inadequate for studies on understanding APPV genetic diversity and evolutionary relationships. This study was intended to investigate the evolutionary relationships and genetic diversity of APPV strains from Guangxi province, Southern China. The results would provide valuable information for research on epidemiological and evolutionary characteristics of APPV in China.

\section{Results}

\section{Detection and amplification of APPV genome from positive samples}

From October 2017 to May 2019, clinical tissue samples (including brain, liver, lymph node and spleen from each piglet) from fifty-three less than one-week old piglets from eighteen pig farms were detected by polymerase chain reaction (PCR) or reverse transcription-PCR (RT-PCR). All the samples were negative for 
CSFV, porcine reproductive and respiratory syndrome virus (PRRSV), porcine circovirus type 2 (PCV2), PCV3, porcine pseudorabies virus (PRV), Japanese encephalitis virus (JEV) and porcine parvovirus (PPV), while 41 samples were positive for APPV (41/53, 77.36\%) (Figure 1).

APPV complete genome was amplified by 8 pairs of specific primers (Table 1) to amplify 8 overlapping fragments encompassing the open reading frame (ORF) and by rapid amplification of CDNA ends (RACE) to amplify 5' untranslated region (UTR) and 3' UTR from APPV positive cDNA samples (Figure 2). After sequencing and assembling, the complete genomic sequences of five APPV strains were obtained and were 11 534-11 565 nucleotides (nt) in full-length, with a 5' UTR of 358-378 nt, followed by a single large ORF and a 3' UTR of 268-279 nt. The ORF was $10908 \mathrm{nt}$ in length, which encoded a polyprotein of 3635 amino acids (aa). The polyprotein was composed of twelve proteins, including four structural proteins (C, $E^{\text {rns }}$, E1 and E2) and eight nonstructural proteins ( ${ }^{\text {pro }}, \mathrm{P} 7, \mathrm{~N} 2, \mathrm{NS} 3, \mathrm{NS} 4 \mathrm{~A}, \mathrm{NS4B}, \mathrm{NS} 5 \mathrm{~A}$ and NS5B) (Table 2). Five APPV strains obtained from this study were named as GX04/2017, GX01-2018, GX02-2018, GX01-2019 and GX02-2019, respectively. The genomic sequences of these identified strains have been submitted to the GenBank database of National Center for Biotechnology Information (NCBI) under accession number MH102210, MH715893, MK453045, MN564752 and MN729215, respectively.

\section{Sequence analyses of APPV genome}

Sequence analysis revealed that the nucleotide identities of the complete genome, ORF, $N^{\text {pro }}, E^{\text {rns }}$ and E2 genes among six APPV strains from Guangxi province, including five strains from this study and one from other researchers, were 83.3-97.5\%, 83.0-98.2\%, 79.6-97.2\%, 80.8-98.4\% and 83.4-96.8\%, respectively; the amino acid identities of ORF, $N^{\text {pro }}, E^{\text {rns }}$ and $E 2$ genes were $91.7-99.1 \%, 81.1-97.8 \%, 89.5-99.0 \%$ and 90.5-96.3\%, respectively (Table 3). The nucleotide identities of the complete genome, ORF, $\mathrm{N}^{\mathrm{pro}}, \mathrm{E}^{\mathrm{rns}}$ and E2 genes among six APPV strains from Guangxi province and the reference strains were $77.7-97.7 \%$, 80.898.6\%, 77.4-99.3\%, 80.3-98.9\% and 79.8-98.9\%, respectively, while the amino acid identities of ORF, Npro, $E^{\text {rns }}$ and E2 genes were $90.6-99.3 \%, 79.4-99.4 \%, 88.1-100 \%$ and $88.0-98.8 \%$, respectively (Table 3).

\section{Phylogenetic analyses of APPV genome}

To investigate the evolutionary relationship and genetic diversity of the APPV strains circulating in Guangxi province, the phylogenetic trees were constructed using the complete genome, $N^{\text {pro }}, E^{\text {rns }}$ and E2 gene sequences of the six strains from Guangxi and other published reference strains available in GenBank (Table 4). All APPV strains could be grouped into four clusters based on the complete genomic sequences (subgroup 01-04 in Figure 3A), $\mathrm{N}^{\text {pro }}$ gene (subgroup N1-N4 in Figure 3B), $\mathrm{E}^{\text {rns }}$ gene (subgroup S1-S4 in Figure 3C) and E2 gene (subgroup E1-E4 in Figure 3D). It was noteworthy that phylogenetic tree based on the complete genomic sequences showed that GX01-2019, APPV GX-Ch2016, GX01-2018 and GX02-2018 strains, together with KU16-2 strain from Korea, Bavaria S5/9 strain from Germany and AUT- 
2016_C strain from Austria, belonged to one branch (subgroup 01); GX04/2017 strain, together with 000515 strain from USA, KU16-6 strain from Korea, belonged to another branch (subgroup 02); GX022019 strain, together with most strains from Guangdong and other provinces in China, belonged to another branch (subgroup 03). The phylogenetic trees based on $\mathrm{N}^{\mathrm{pro}}, \mathrm{E}^{\mathrm{rns}}$ and $\mathrm{E} 2$ gene sequences showed quite similar topology to that of complete genome with a little disagreement. The above results showed that all six strains from Guangxi province existed a high degree of genetic diversity and obviously different evolutionary relationship.

\section{Evolution analyses of APPV epidemic strains}

To evaluate the recombination events of APPV strains during the process of evolution, the complete genomic sequences of 58 APPV strains form different countries available in GenBank (Table 4) were analyzed using Recombination Detection Program 4 (RDP4) and Simplot 3.5.1. The results showed that there existed recombination detection signs in six strains, including GD-BZ01-2018 (MH493896), GDDH01-2018 (MH493895), YN01/2017 (MH378079), JX-JM01-2018A01 (MG792803), GD-LN-2017.04 (MK216753) and GD2 (KX950763) (Table 5), and all of them came from China. No sign of recombination was detected in all six strains from Guangxi province (Figure 4).

Evolutionary estimation based on the complete genome, $N^{\text {pro }}, E^{\text {rns }}$ and $E 2$ gene sequences of 58 APPV strains form different countries was conducted by Bayesian analysis. The results indicated that APPV genomic sequences evolved at a mean rate of $1.37 \times 10^{-4}\left(95 \%\right.$ HPD $\left.5.12 \times 10^{-6}-3.02 \times 10^{-4}\right)$ substitutions/site/year ( $\mathrm{s} / \mathrm{s} / \mathrm{y})$, and the mean rates of molecular evolution of $\mathrm{N}^{\text {pro }}, \mathrm{E}^{\mathrm{rns}}$ and $\mathrm{E} 2 \mathrm{gene}$ sequences were $1.00 \times 10^{-4}\left(8.47 \times 10^{-5}-1.17 \times 10^{-4}\right), 1.56 \times 10^{-4}\left(9.81 \times 10^{-5}-2.20 \times 10^{-4}\right)$ and $1.01 \times 10^{-4}\left(8.17 \times 10^{-}\right.$ $\left.5-1.22 \times 10^{-4}\right) \mathrm{s} / \mathrm{s} / \mathrm{y}$, respectively. Bayesian inferences (BI) estimated that the tMRCA of complete genomes of APPV epidemic strains existed 1700.5 (95\% HPD 228.4-4654.5) years ago, and the tMRCAs of $N^{\text {pro, }}$ $E^{\text {rns }}$ and E2 gene sequences were 1495.5 (1404.3-1597.4), 1390.8 (821.9-2032.2) and 1823.0 (1723.21918.1) years ago, respectively.

\section{Discussion}

CT has been classified as type $A$ and type $B$, of which type $A$ is associated with variable hypomyelination

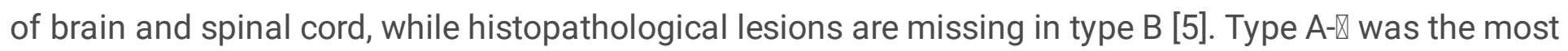
common CT in newborn piglets, which characterized by generalized shaking and varying degrees of absence of myelin sheath in brain and spinal cord [9]. The clinical presentation of APPV-infected pigs was characterized by CT type A-II in piglets, while adult pigs might become persistent carriers and shedders [5, 7]. It could occur as a sporadic disease affecting single litters or as an outbreak over several weeks affecting multiple litters, and the losses of 2.5 piglets per sow and a $10 \%$ drop in pig reproductive 
performance was occurred on a farm in Austria [7]. CT in newborn piglets was first reported in 1922, but the causative agent has always been a mystery. Viral agents responsible for type A- $\triangle \mathrm{CT}$ had been seeking for decades until APPV was identified in USA and other countries in 2015 [3-17]. APPV have been detected in serum, thymus, peripheral lymphoid organs (spleen, tonsil, submaxillary lymph node and inguinal lymph node), nervous system (brain stem, brain, and cerebellum), digestive system (duodenum) and semen $[1,8,29,30,31,32]$, which indicated that this virus has widespread tissue tropism. However, it was very difficult for this virus to culture and identify. Many researchers have attempted to seek appropriate cell lines to culture and acquire higher titer of APPV for identification and characterization, but unfortunately, no one has been successful until now because this virus could not replicate in the selected cell lines or the viral titer was too low $[1,5,6,7,26,29]$. Therefore, all the APPV sequences obtained until now were directly amplified, cloned and sequenced from positive serum and tissue samples $[1,5,7,18,19,21]$, and the complete genomic sequences of five APPV strains obtained in this study were determined in the same way. Finally, a total of six APPV strains available in GenBank until February 29, 2020, including five strains from this study and one strain from other researchers [18], were used to analyze the evolution and genetic diversity of APPV epidemic strains in Guangxi province, Southern China in this study.

Sequence analysis revealed that six APPV strains from Guangxi province shared $83.3-97.5 \%$ nucleotide identity with one another, and shared 77.7-97.7\% nucleotide identity with other reference strains available in GenBank, showing that the most genetic distance reached 22.3\%. Similar results have been reported by other studies. The complete polyprotein coding sequences of German APPV demonstrated to be highly variable from different domestic regions with genetic variability of $81-87 \%$ among the APPV isolates, and shared $88.2 \%$ nucleotide identity with the APPV sequences from USA [6, 33]. The genomic sequence of APPVs from Austria shared $90 \%$ nucleotide identity with those from USA and $92 \%$ nucleotide identity with those from Germany [7]. Two Chinese APPV strains named as GD1 and GD2 from Guangdong province shared $83.1 \%-83.5 \%$ nucleotide identity with other APPV strains from different countries [14], and other three APPV strains also from Guangdong province exhibited only $80.5 \%-84.1 \%$ nucleotide identity to reference strains available in GenBank [34], while APPV_GD strain shared the highest nucleotide identity of $93.5 \%$ to Bavaria_S5/9 strain from Germany [31]. Those APPV strains derived from Guangdong province analyzed in this study shared $80.8 \%-99.6 \%$ nucleotide identity to one another. These results indicated that there existed high genetic diversity of current APPV strains from different countries around the world. Phylogenetic analysis revealed that all APPV strains from Guangxi province distributed in three subgroups (01-03). GX01-2018, GX02-2018, APPV_CH-GX_2016 and GX012019 strains together with NL1, Bavaria_S5/9 and AUT-2016_C reference strains from European belonged to 01 subgroup. GX04/2017 strain together with 000515 strain from USA belonged to 02 subgroup. GX02-2019 strain together with most strains from Guangdong province belonged to 03 subgroup. Interestingly, we also found that APPV strains except for APPV_GD from Guangdong province belonged to an independent subgroup, while APPV_GD strain together with strains from Europe and Korea belonged to another subgroup. One study reported that the genomic sequences of 14 APPV strains from Guangdong province shared $80.6-99.8 \%$ nucleotide identities and distributed in three well-defined clades 
including a newly emerging branch in China, which showed high genetic diversity among these strains [23]. These phenomena might due to the multiple sources of stud pigs and boar semen in China, which not only came from North America countries such as USA and Canada, but also came from European countries such as Denmark, France and Germany, and APPV might spread into China through the infected pigs and contaminated semen from America and Europe. The results suggested that not only within a country but also within an area, such as Guangxi Province, APPV exhibited a high genetic diversity among viral strains.

Recombination analysis of six APPV strains from Guangxi province and other 52 reference strains available in GenBank showed that there existed recombination in six reference strains and no recombination in all six strains from Guangxi province. Interestingly, all the recombinant strains came from China, and the possible reason might be contributed to multiple sources of APPV around the world, and there were more chances to take place genetic recombination. An identical result was confirmed by the SimPlot software. Similarity analysis of APPV genomes indicated that there existed high variation regions in whole genomic sequences, which would be a big challenge for molecular diagnosis and epidemiological investigation of APPV. It was noteworthy that the similarity relationship of GX01-2018 strain was close to NL1 and AUT-2016_C strains from Europe, while GX04/2017 strain presented relative higher similarity to 000515 from North America [21], suggesting again that there existed high genetic diversity and variation of APPV strains from Guangxi province.

To our knowledge, this is the first report on estimation of the mean rate of molecular evolution and dates of the tMRCA about APPV. The results revealed that APPV genomic sequences evolved at an evolutionary rate of $1.37 \times 10^{-4}\left(95 \% \mathrm{HPD}: 5.12 \times 10^{-6}-3.02 \times 10^{-4}\right) \mathrm{s} / \mathrm{s} / \mathrm{y}$, while $\mathrm{N}^{\mathrm{pro}}, \mathrm{E}^{\mathrm{rns}}$ and $\mathrm{E} 2$ gene sequences evolved at a rate of $1.00 \times 10^{-4}\left(8.47 \times 10^{-5}-1.17 \times 10^{-4}\right), 1.56 \times 10^{-4}\left(9.81 \times 10^{-5}-2.20 \times 10^{-4}\right)$ and 1.01 $\times 10^{-4}\left(8.17 \times 10^{-5}-1.22 \times 10^{-4}\right) \mathrm{s} / \mathrm{s} / \mathrm{y}$, respectively. The results indicated that tMRCA of APPV genome was 1700.5 (95\% HPD: 228.4-4654.5) years ago, while tMRCA of $\mathrm{N}^{\text {pro }}, \mathrm{E}^{\text {rns }}$ and E2 genes were estimated as 1495.5 (1404.3-1597.4), 1390.8 (821.9-2032.2) and 1823.0 (1723.2-1918.1) years ago, respectively. As $\mathrm{N}^{\text {pro }}$ is unique to the genus Pestivirus and is a non-structural autoprotease [35] and $\mathrm{N}^{\text {pro }}$ sequence had only $9 \%-18 \%$ pairwise amino acid identity to other $\mathrm{N}^{\text {pro }}$ sequences [1], which suggest that $\mathrm{N}^{\text {pro }}$ gene might be highly variable constitution of APPV genome. The E2 protein is immunodominant and possesses neutralization epitopes and exhibits the greatest amount of diversity [36, 37], which means E2 gene might plays an important role in evolution of the virus under selective pressure. Some previous studies had been done on the evolution of other pestiviruses. A mean substitution rate of $1.4 \times 10^{-3} \mathrm{~s} / \mathrm{s} / \mathrm{y}$ was found across both BVDV1 and BVDV2 and the tMRCA was estimated to be 1498 (95\% HPD: 1182-1750) years ago [38]; the estimated mean evolutionary rate of the $5^{\prime}$ UTR BDV sequences was $2.9 \times 10^{-3} \mathrm{~s} / \mathrm{s} / \mathrm{y}(95 \%$ HPD: $1.5 \times 10^{-3}-4.6 \times 10^{-3}$ ) [39]. Evolutionary analysis on the full-length E2 sequences showed that CSFV from different countries had an evolution rate of $3.2 \times 10^{-4} \mathrm{~s} / \mathrm{s} / \mathrm{y}$ and the origin of CSFV was estimated to be the mid-1500s [40], and the mean substitution rate for Korean CSFV from 1987-2017 was estimated as $2.21 \times 10^{-3} \mathrm{~s} / \mathrm{s} / \mathrm{y}\left(95 \% \mathrm{HPD}: 1.70 \times 10^{-3}-2.76 \times 10^{-3}\right)$ and the tMRCA was 1901 years ago (95\% HPD: 1865-1933) [41], while analysis on the whole CSFV genome showed that CSFV has evolved 
at a rate of $1.03 \times 10^{-4} \mathrm{~s} / \mathrm{s} / \mathrm{y}\left(95 \%\right.$ HPD: $\left.2.03 \times 10^{-4}-2.61 \times 10^{-4}\right)$ and the tMRCA appeared 2770.2 years ago (95\% HPD: 223.5-8611.6) [42]. As for other highly variable RNA virus, such as PRRSV and swine influenza virus (SIV), some studies reported that the evolutionary rate of PRRSV genome ranged from $1.98 \times 10^{-3}$ to $3.29 \times 10^{-3} \mathrm{~s} / \mathrm{s} / \mathrm{y}[43,44]$ and that of SIV HA gene ranged from $1.03 \times 10^{-3}$ to $3.18 \times 10^{-}$

${ }^{3} \mathrm{~s} / \mathrm{s} / \mathrm{y}[45,46]$. These results showed that the evolutionary rate of APPV was similar to that of CSFV, and lower than those of other RNA viruses reported in the publications. This study might provide complementary reference for evolutionary information of APPV in the genus Pestivirus.

\section{Conclusions}

In this study, the complete genomic sequences of five APPV strains were obtained by amplification, sequence and assembly from positive clinical tissue samples from neonatal piglets with CT in Guangxi province, Southern China. The six APPV strains from Guangxi province, including five strains from this study and one strain from other researchers, shared $83.3 \%-97.5 \%$ nucleotide identity of complete genome and $91.7 \%-99.1 \%$ amino acid identity of open reading frame (ORF), and distributed in three subgroups in phylogenetic trees based on complete genome, $\mathrm{N}^{\text {pro }}, \mathrm{E}^{\mathrm{rns}}$ and $\mathrm{E} 2$ gene sequences, respectively, which indicated that APPV strains from Guangxi province existed high degree of genetic diversity and variation. The results provided valuable information on epidemiological and evolutionary characteristics of APPV in Southern China.

\section{Methods}

\section{Collection of clinical samples}

From October 2017 to May 2019, newborn piglets less than one-week old showing clinical manifestations of CT and splayed legs were reported from eighteen farms in Guangxi province. Piglets presented CT soon after birth and some of them died within a week. Fifty-three piglet samples, including brain, liver, spleen and lymph node from each dead or sick piglet, were collected with written consent of the veterinarians and the farm owners and submitted to our lab for diagnostic investigation. Identification of CT-associated viral pathogens were detected by PCR or RT-PCR for APPV, CSFV, PRRSV, PCV2, PCV3, PRV, JEV and PPV. The primers and reaction condition profiles for detection of these viral pathogens by PCR or RT-PCR are available upon request.

\section{Detection and sequence determination of APPV genome}

The tissue samples of brain, liver, spleen and lymph nodes were homogenized in phosphate-buffered saline solution (PBS, pH7.2) using a blender (Tianlong, Xi'an, China) followed by shaking with small glass beads for $5 \mathrm{~min}$. The homogenized material $(10 \%, \mathrm{~W} / \mathrm{V})$ was then freeze-thawed three times, centrifuged at $10000 \times \mathrm{g}$ for $10 \mathrm{~min}$ at $4^{\circ} \mathrm{C}$ and used to determine APPV presence by RT-PCR. Viral RNA was extracted 
from the supernatant using MiniBEST RNA/DNA Extraction Kit (TaKaRa, Dalian, China) and reverse transcribed to cDNA using PrimeScript $\ 1$ st Strand cDNA Synthesis Kit (TaKaRa, Dalian, China) according to the manufacturer's instructions. Then, the following pair of primers (5'UTR-forward: $5^{\prime}-$

TGGGGGAAAGGGGTTAACCAG-3' and 5'UTR-reverse: 5'-ATCCGCCGGCACTCTATCAAG-3') was used to amplify 275 bp fragment of 5 ' untranslated region (UTR) by RT-PCR. Briefly, amplification was carried out in a total volume of $25 \mu \mathrm{L}$ containing: $12.5 \mu \mathrm{L} 2 \times$ Taq PCR MasterMix (TaKaRa, Dalian, China), $5 \mu \mathrm{L}$ of cDNA sample, $0.5 \mu \mathrm{L}$ of both primers $(25 \mathrm{pmol} / \mu \mathrm{L})$ and $6.5 \mu \mathrm{L}$ sterilized distill water. The reaction was done under the following condition: 1 cycle of $94^{\circ} \mathrm{C}$ for $3 \mathrm{~min} ; 35$ cycles of denaturation at $94^{\circ} \mathrm{C}$ for $30 \mathrm{~s}$, annealing at $56^{\circ} \mathrm{C}$ for $30 \mathrm{~s}$, and elongation at $72^{\circ} \mathrm{C}$ for $30 \mathrm{~s}$; and 1 cycle at $72^{\circ} \mathrm{C}$ for $10 \mathrm{~min}$. The amplified products were analyzed by electrophoresis in a $2 \%$ agarose gel, stained with ethidium bromide $(0.5 \mathrm{~g} / \mathrm{mL})$ in TBE buffer ( $\mathrm{pH} 8.4)$. The cDNAs from five APPV positive samples were used for amplifying the complete genome of APPV.

To determine the complete genome, RT-PCR was used with 8 pairs of specific primers (Table 1) to amplify 8 overlapping fragments encompassing the open reading frame (ORF) of APPV strains. PCR was carried out in a total volume of $50 \mu \mathrm{L}$ containing: $25 \mu \mathrm{L} 2 \times$ Gflex PCR Buffer $\left(\mathrm{Mg}^{2+}\right.$, dNTP plus) (TaKaRa, Dalian, China), $5 \mu \mathrm{L}$ cDNA sample, $1 \mu \mathrm{L}$ of both primers (10 pmol/ $\mu \mathrm{L}), 1 \mu \mathrm{L}$ TKs Gflex DNA Polymerase (1.25 U/ $\mu \mathrm{L})$ (TaKaRa, Dalian, China) and $17 \mu \mathrm{L}$ sterilized distill water. PCR was performed as follows: 1 cycle of $94^{\circ} \mathrm{C}$ for $3 \mathrm{~min} ; 35$ cycles of $98^{\circ} \mathrm{C}$ for $10 \mathrm{~s}, 52^{\circ} \mathrm{C}$ for $15 \mathrm{~s}$, and $68^{\circ} \mathrm{C}$ for $2 \mathrm{~min}$; and a final extension of $72^{\circ} \mathrm{C}$ for $10 \mathrm{~min}$. The $5^{\prime}$ UTR and $3^{\prime}$ UTR were obtained by rapid amplification of cDNA ends (RACE) using SMARTer RACE 5'/3' Kit (TaKaRa, Dalian, China) according to the manufacturer's instructions. The amplified fragments were purified and cloned into a pMD18-T vector (TaKaRa, Dalian, China), and sequenced with an $\mathrm{ABI} 3730 \mathrm{XL}$ sequencer (TaKaRa, Dalian, China). After assembling the sequences of above-mentioned fragments with Lasergene SeqMan Program (DNASTAR, USA), the complete genomic sequences of five APPV strains from Guangxi province were obtained and named as GX04/2017, GX012018, GX02-2018, GX01-2019 and GX02-2019, respectively. The genomic sequences of these identified strains have been submitted to the GenBank database under accession number MH102210, MH715893, MK453045, MN564752 and MN729215, respectively.

\section{Evolution and phylogenetic analysis of APPV genome}

Nucleotide and predicted amino acid identity analysis was calculated using MegAlign program of the DNAStar package (DNASTAR, USA). To determine the relationship between current and previously published APPV strains, the reference strains from different geographic locations and collection date were downloaded from GenBank database at NCBI (Table 4). The best fitting nucleotide substitution model of APPV genome, $N^{\text {pro }}, E^{\text {rns }}$ and E2 genes was firstly determined. General Time Reversible parameter plus the Gamma Distributed with Invariant sites $(G T R+G+I)$ for APPV genome, $N^{\text {pro }}, E^{\text {rns }}$ and E2 genes was selected as the best-fit model using ModelFinder program of IQ-TREE online software (http://iqtree.cibiv.univie.ac.at/). Maximum Likelihood analysis based on complete genome, $\mathrm{N}^{\text {pro }}$, $\mathrm{E}^{\text {rns }}$ and 
E2 gene sequences were implemented in MEGA X under the following parameters: model of nucleotide substitution, $\mathrm{GTR}+\mathrm{G}+\mathrm{l}$; initial tree, $\mathrm{NJ} / \mathrm{BioNJ}$; bootstrap method test with 1000 replicates; Gamma distributed; number of substitution rate categories. Phylogenetic trees were constructed by MEGA X. The APPV complete genomic sequences were analyzed using Recombination Detection Program 4 (RDP4) and then confirmed with SimPlot software (version 3.5.1). Bayesian inference analysis was performed using BEAST software package (version 1.10.4), the times of the most recent common ancestor (tMRCA) and mean rate of molecular evolution were calculated by BEAST software, the output of which was explored by program Tracer (version 1.7.4), and the statistical uncertainties were summarized in $95 \%$ highest probability density (HPD) intervals.

\section{Abbreviations}

APPV

Atypical porcine pestivirus; CSFV:Classical swine fever virus; JEV:Japanese encephalitis virus; ORF:Open reading frame; PCR:Polymerase chain reaction; PCV2:Porcine circovirus type 2; PCV3:Porcine circovirus type 3; RT-PCR:Reverse transcription-PCR; PPV:Porcine parvovirus; PRRSV:Porcine reproductive and respiratory syndrome virus; PRV:Porcine pseudorabies virus; UTR:Untranslated region

\section{Declarations}

\section{Acknowledgments}

Not applicable.

\section{Funding}

This work was funded by Guangxi Science and Technology Bureau (AA17204057) and Guangxi Agricultural and Rural Bureau (2019Z54), China. The founder had no role in the study design, sample collection, detection, sequencing, analysis and interpretation, or the manuscript preparation.

\section{Availability of data and materials}

All data were included in the manuscript as tables and figures. The genomic sequences of GX04/2017, GX01-2018, GX02-2018, GX01-2019 and GX02-2019 strains obtained in this study were submitted to the GenBank database under the accession numbers MH102210, MH715893, MK453045, MN564752 and MN729215, respectively and the reference strains used in this study are listed in Table 4. 


\section{Authors' contributions}

SK, XS, ZJ and LH conducted the experiments and analyzed the data. YY and QS assisted with sample preparation and experiments. CZ, LW and FS shared ideas and discussed the research data. SK and SH contributed to supervision, had the idea for the project and directed the research. All authors have read and approved the final manuscript.

\section{Competing interest}

The authors declare that they have no competing interests.

\section{Ethics approval and consent to participate}

This study was approved by the Animal Ethics Committee of Guangxi University and the Animal Ethics Committee of Guangxi Center for Animal Disease Control and Prevention (P-2017-02), Nanning, China. All porcine clinical samples used in this study were collected with written consent of the veterinarians and the farm owners. All procedures were carried out in strict accordance with the Animal Ethics Procedures and Guidelines of the People's Republic of China. All the Animal protocols in this study were conducted according to the approved procedures.

\section{Consent for publication}

Not applicable.

\section{References}

1. Hause BM, Collin EA, Peddireddi L, Yuan F, Chen Z, Hesse RA, Gauger PC, Clement T, Fang Y, Anderson G: Discovery of a novel putative atypical porcine pestivirus in pigs in the USA. J Gen Virol. 2015;96(10):2994-8.

2. Pan S, Mou C, Chen Z: An emerging novel virus: Atypical porcine pestivirus (APPV). Rev Med Virol. 2019;29(1):e2018.

3. Gatto IRH, Sonalio K, de Oliveira LG: Atypical porcine pestivirus (APPV) as a new species of pestivirus in pig production. Front Vet Sci. 2019;6:35. 
4. Arruda BL, Arruda PH, Magstadt DR, Schwartz KJ, Dohlman T, Schleining JA, Patterson AR, Visek CA, Victoria JG: Identification of a divergent lineage porcine pestivirus in nursing piglets with congenital tremors and reproduction of disease following experimental inoculation. PLoS One. 2016;11(2):e0150104.

5. de Groof A, Deijs M, Guelen L, van Grinsven L, van Os-Galdos L, Vogels W, Derks C, Cruijsen T, Geurts V, Vrijenhoek M, et al: Atypical porcine pestivirus: A possible cause of congenital tremor type A-Il in newborn piglets. Viruses. 2016;8(10).

6. Postel A, Hansmann F, Baechlein C, Fischer N, Alawi M, Grundhoff A, Derking S, Tenhundfeld J, Pfankuche VM, Herder V, et al: Presence of atypical porcine pestivirus (APPV) genomes in newborn piglets correlates with congenital tremor. Sci Rep. 2016;6:27735.

7. Schwarz L, Riedel C, Hogler S, Sinn LJ, Voglmayr T, Wochtl B, Dinhopl N, Rebel-Bauder B, Weissenbock $H$, Ladinig $A$, et al: Congenital infection with atypical porcine pestivirus (APPV) is associated with disease and viral persistence. Vet Res. 2017;48(1):1.

8. Munoz-Gonzalez S, Canturri A, Perez-Simo M, Bohorquez JA, Rosell R, Cabezon O, Segales J, Domingo M, Ganges L: First report of the novel atypical porcine pestivirus in Spain and a retrospective study. Transbound Emerg Dis. 2017;64(6):1645-9.

9. Mosena ACS, Weber MN, da Cruz RAS, Cibulski SP, da Silva MS, Puhl DE, Hammerschmitt ME, Takeuti KL, Driemeier D, de Barcellos D, et al: Presence of atypical porcine pestivirus (APPV) in Brazilian pigs. Transbound Emerg Dis. 2018;65(1):22-6.

10. Denes L, Biksi I, Albert M, Szeredi L, Knapp DG, Szilasi A, Balint A, Balka G: Detection and phylogenetic characterization of atypical porcine pestivirus strains in Hungary. Transbound Emerg Dis. 2018;65(6):2039-42.

11. Williamson S: Congenital tremor associated with atypical porcine pestivirus. Vet Rec. 2017;180(2):42-3.

12. Fanny G. Dessureault MC, Chantale Provost, Carl A. Gagnon: First report of atypical porcine pestivirus in piglets with congenital tremor in Canada. Can Vet J. 2018;59(4):429-32.

13. Blomstrom AL, Fossum C, Wallgren P, Berg M: Viral metagenomic analysis displays the co-infection situation in healthy and PMWS affected pigs. PLoS One. 2016;11(12):e0166863.

14. Zhang K, Wu K, Liu J, Ge S, Xiao Y, Shang Y, Ning Z: Identification of atypical porcine pestivirus infection in swine herds in China. Transbound Emerg Dis. 2017;64(4):1020-3.

15. Kim S, Jeong C, Yoon S, Lee K, Yang M, Kim B: Detection of atypical porcine pestivirus (APPV) from a case of congenital tremor in Korea. Korean J Vet Serv. 2017;40:209-13.

16. Kaufmann C, Stalder H, Sidler X, Renzullo S, Gurtner C, Grahofer A, Schweizer M: Long-term circulation of atypical porcine pestivirus (APPV) within Switzerland. Viruses. 2019;11(7).

17. Sozzi E, Salogni C, Lelli D, Barbieri I, Moreno A, Alborali GL, Lavazza A: Molecular survey and phylogenetic analysis of atypical porcine pestivirus (APPV) identified in swine and wild boar from Northern Italy. Viruses. 2019;11(12). 
18. Zhang H, Wen W, Hao G, Hu Y, Chen H, Qian P, Li X: Phylogenetic and genomic characterization of a novel atypical porcine pestivirus in China. Transbound Emerg Dis. 2018;65(1):e202-4.

19. Shen H, Liu X, Zhang P, Wang L, Liu Y, Zhang L, Liang P, Song C: Identification and characterization of atypical porcine pestivirus genomes in newborn piglets with congenital tremor in China. J Vet Sci. 2018;19(3):468-71.

20. Wu S, Wang Z, Zhang W, Deng S: Complete genome sequence of an atypical porcine pestivirus isolated from Jiangxi province, China. Genome Announc. 2018;6(24).

21. Pan S, Yan Y, Shi K, Wang M, Mou C, Chen Z: Molecular characterization of two novel atypical porcine pestivirus (APPV) strains from piglets with congenital tremor in China. Transbound Emerg Dis. 2019;66(1):35-42.

22. Zhou K, Yue H, Tang C, Ruan W, Zhou Q, Zhang B: Prevalence and genome characteristics of atypical porcine pestivirus in southwest China. J Gen Virol. 2019;100(1):84-8.

23. Yan XL, Li YY, He LL, Wu JL, Tang XY, Chen GH, Mai KJ, Wu RT, Li QN, Chen YH, et al: 12 novel atypical porcine pestivirus genomes from neonatal piglets with congenital tremors: A newly emerging branch and high prevalence in China. Virology. 2019;533:50-8.

24. Xie Y, Wang X, Su D, Feng J, Wei L, Cai W, Li J, Lin S, Yan H, He D: Detection and genetic characterization of atypical porcine pestivirus in piglets with congenital tremors in Southern China. Front Microbiol. 2019;10:1406.

25. Smith DB, Meyers G, Bukh J, Gould EA, Monath T, Scott Muerhoff A, Pletnev A, Rico-Hesse R, Stapleton JT, Simmonds P, et al: Proposed revision to the taxonomy of the genus Pestivirus, family Flaviviridae. J Gen Virol. 2017;98(8):2106-12.

26. Cagatay GN, Antos A, Meyer D, Maistrelli C, Keuling O, Becher P, Postel A: Frequent infection of wild boar with atypical porcine pestivirus (APPV). Transbound Emerg Dis. 2018;65(4):1087-93.

27. Gatto IRH, Arruda PH, Visek CA, Victoria JG, Patterson AR, Krull AC, Schwartz KJ, de Oliveira LG, Arruda BL: Detection of atypical porcine pestivirus in semen from commercial boar studs in the United States. Transbound Emerg Dis. 2018;65(2):e339-43.

28. Michelitsch A, Dalmann A, Wernike K, Reimann I, Beer M: Seroprevalences of newly discovered porcine pestiviruses in German pig farms. Vet Sci. 2019;6(4).

29. Gatto IRH, Harmon K, Bradner L, Silva P, Linhares DCL, Arruda PH, de Oliveira LG, Arruda BL: Detection of atypical porcine pestivirus in Brazil in the central nervous system of suckling piglets with congenital tremor. Transbound Emerg Dis. 2018;65(2):375-80.

30. Postel A, Meyer D, Cagatay GN, Feliziani F, De Mia GM, Fischer N, Grundhoff A, Milicevic V, Deng MC, Chang $\mathrm{CY}$, et al: High abundance and genetic variability of atypical porcine pestivirus in pigs from Europe and Asia. Emerg Infect Dis. 2017;23(12):2104-7.

31. Yuan J, Han Z, Li J, Huang Y, Yang J, Ding H, Zhang J, Zhu M, Zhang Y, Liao J, et al: Atypical porcine pestivirus as a novel type of pestivirus in pigs in China. Front Microbiol. 2017;8:862.

32. Liu J, Li Z, Ren X, Li H, Lu R, Zhang Y, Ning Z: Viral load and histological distribution of atypical porcine pestivirus in different tissues of naturally infected piglets. Arch Virol. 2019;164(10):2519-23. 
33. Beer M, Wernike K, Drager C, Hoper D, Pohlmann A, Bergermann C, Schroder C, Klinkhammer S, Blome S, Hoffmann B: High prevalence of highly variable atypical porcine pestiviruses found in Germany. Transbound Emerg Dis. 2017;64(5):e22-6.

34. Zhang X, Dai R, Li Q, Zhou Q, Luo Y, Lin L, Bi Y, Chen F: Detection of three novel atypical porcine pestivirus strains in newborn piglets with congenital tremor in southern China. Infect Genet Evol, 2019;68:54-7.

35. Stark G, Meyers G, Rümenapf T, Thief HJ: Processing of pestivirus polyprotein: cleavage site between autoprotease and nucleocapsid protein of classical swine fever virus. J Virol. 1993;67(12):7088-95.

36. Ridpath JF, Bolin SR: Comparison of the complete genomic sequence of the border disease virus, BD31, to other pestiviruses. Virus Res. 1997;50(2):237-43.

37. Tijssen P, Pellerin C, Lecomte J, van den Hurk J: Immunodominant E2 (gp53) sequences of highly virulent bovine viral diarrhea group II viruses indicate a close resemblance to a subgroup of border disease viruses. Virology. 1996;217(1):356-61.

38. Chernick A, van der Meer F: Evolution of bovine viral diarrhea virus in Canada from 1997 to 2013. Virology. 2017;509:232-8.

39. Lzzgo C, Ebranati E, Cabezón O, Fernández-Sirera L, Lavin S, Rosell R, Veo C, Rossi L, Cavallero S, Lanfranchi P, et al: Spatial and temporal phylogeny of border disease virus in Pyrenean chamois (Rupicapra p. pyrenaica). PLoS One. 2016;11(12): e0168232.

40. Garrido Haro AD, Barrera Valle M, Acosta A, F JF: Phylodynamics of classical swine fever virus with emphasis on Ecuadorian strains. Transbound Emerg Dis. 2018;65(3):782-90.

41. An DJ, Lim SI, Choe S, Kim KS, Cha RM, Cho IS, Song JY, Hyun BH, Park BK: Evolutionary dynamics of classical swine fever virus in South Korea: 1987-2017. Vet Microbiol. 2018;225:79-88.

42. Kwon T, Yoon SH, Kim KW, Caetano-Anolles K, Cho S, Kim H: Time-calibrated phylogenomics of the classical swine fever viruses: genome-wide bayesian coalescent approach. PLoS One. 2015;10(3):e0121578.

43. Song J, Shen D, Cui J, Zhao B: Accelerated evolution of PRRSV during recent outbreaks in China. Virus Genes. 2010;41(2):241-5.

44. Yoon SH, Kim H, Kim J, Lee HK, Park B, Kim H: Complete genome sequences of porcine reproductive and respiratory syndrome viruses: perspectives on their temporal and spatial dynamics. Mol Biol Rep. 2013;40:6843-53.

45. Wei K, Tang X, Li Y: Genome-scale phylodynamics and evolution analysis of global H7N7 influenza viruses. Vet Microbiol. 2016;193:83-92.

46. Al Khatib HA, Al Thani AA, Yassine HM: Evolution and dynamics of the pandemic H1N1 influenza hemagglutinin protein from 2009 to 2017. Arch Virol. 2018;163(11):3035-49.

\section{Tables}


Table 1 Primers used for amplifying the complete genome of APPV

\begin{tabular}{|c|c|c|c|c|}
\hline Primer & Sequence $\left(5^{\prime} \rightarrow 3^{\prime}\right)$ & Target position & Product/bp & Template \\
\hline 5'RACE & 5'-CGCGGATCCACAGCCTACTGATGATCAGTCGATG-3' & l & 336 & 000515 \\
\hline 5'-Inner & 5'-CCGGCACTCTATCAAGCAGTAAGGTC-3' & 311-336 & & \\
\hline P1-F & 5'-GCATAATGCTTTGATTGGCTGCAT-3' & $1-24$ & 1432 & \\
\hline P1-R & 5'-GGCTTGTRTCTATCATTCCCAG-3' & 1411-1432 & & \\
\hline P2-F & 5'-AAGGTYCAGTGGTTCTTAAAGG-3' & $1258-1279$ & 2114 & \\
\hline P2-R & 5'-AGRAAGCTCAAGGCTACTGGAC-3' & $3350-3371$ & & \\
\hline P3-F & 5'-TGTGGAAAATGGACTGGACAGA-3' & $3239-3260$ & 1902 & \\
\hline P3-R & 5'-ACCTCATRAAGCGRCAGACACT-3' & $5119-5140$ & & \\
\hline P4-F & 5'-GAATTGGCAGATATGCGGAGG-3' & $4969-4989$ & 1783 & \\
\hline P4-R & 5'-CCTGATGYTTCCTCAAGTAYTG-3' & $6730-6751$ & & \\
\hline P5-F & 5'-GACCAYCAACTGAGGCAACTAC-3' & $6601-6622$ & 1627 & \\
\hline P5-R & 5'-TCTTGGATCCACGRTGTGCTTT-3' & $8206-8227$ & & \\
\hline P6-F & 5'-GCCAAGTGGCCATAGGYAAAGT-3' & $8102-8123$ & 2022 & \\
\hline P6-R & 5'-ACTGAGCCCAATCTGCACTBAC-3' & $10102-10123$ & & \\
\hline P7-F & 5'-AGAAACCACGTGTGATACAGT-3' & $9962-9982$ & 1495 & \\
\hline P7-R & 5'-CAAGTATTTACAACAACCCCAT-3' & $11435-11456$ & & \\
\hline P8-F & 5'-AGAAACCACGTGTGATACAGT-3' & $9962-9982$ & 1602 & \\
\hline P8-R & 5'-TGGCCCCCTTGCTTCATCTAGATC-3' & 11540-11564 & & \\
\hline 3'-Inner & 5'-AAAGACGAGCCAGCGGTTAGTTGA-3' & $11002-11025$ & 560 & \\
\hline 3'RACE & 5'-CGCGGATCCTCCACTAGTGATTTCACACTATAGC-3' & l & & \\
\hline
\end{tabular}

Table 2 The genome organization of APPV strains obtained in this study

\begin{tabular}{lllll}
\hline Genome & Coded protein & Position & Nucleotide (nt) & Amino acid (aa) \\
\hline 5' UTR & I & $1-378$ & $358-378$ & $/$ \\
ORF & N ${ }^{\text {pro }}$ & $379-918$ & 540 & 180 \\
& C & $919-1251$ & 333 & 111 \\
& E $^{\text {rns }}$ & $1252-1881$ & 630 & 210 \\
& E1 & $1882-2478$ & 597 & 199 \\
& E2 & $2479-3201$ & 723 & 241 \\
& P7 & $3202-3393$ & 192 & 64 \\
& NS2 & $3394-4335$ & 942 & 314 \\
& NS3 & $4336-6396$ & 2061 & 687 \\
& NS4A & $6397-6597$ & 201 & 67 \\
& NS4B & $6598-7614$ & 1017 & 339 \\
& NS5A & $7615-9030$ & 1416 & 472 \\
& NS5B & $9031-11286$ & 2253 & 751 \\
\hline
\end{tabular}

Note: The complete genomic sequence of GX02-2018 (MK453045), GX01-2019 (MN564752) and GX02-2019 (MN729215) strains was 11564 nt in fulllength, with a 5' UTR of 378 nt, followed by an ORF of 10908 nt and a 3' UTR of 278 nt. The complete genomic sequence of GX04/2017 (MH102210) /GX01-2018 (MH715893) was 11 534/11 565 nt in full-length, with a 5' UTR of 358/378 nt, followed by an ORF of 10908 nt and a 3' UTR of 268/279 nt

Table 3 Nucleotide and amino acid identity (\%) of identified strains and reference strains of APPV 


\begin{tabular}{|c|c|c|c|c|c|c|c|c|c|c|}
\hline \multirow[t]{2}{*}{ Strain } & \multicolumn{5}{|c|}{$\begin{array}{l}\text { Nucleotide and amino acid identity among } \\
\text { identified strains }\end{array}$} & \multicolumn{5}{|c|}{$\begin{array}{l}\text { Nucleotide and amino acid identity between identified strains and } \\
\text { reference strains }\end{array}$} \\
\hline & Genome & ORF & $\mathrm{N}^{\text {pro }}$ & $E^{\text {rns }}$ & E2 & Genome & ORF & $\mathrm{N}^{\text {pro }}$ & $E^{\text {rns }}$ & E2 \\
\hline APPV_GX- & 83.3- & 83.5- & $79.8-97.2$ & 82.4-98.4 & 83.4-96.8 & $79.0-97.7$ & $81.3-97.8$ & $77.4-97.2$ & $80.6-98.9$ & $81.1-97.4$ \\
\hline CH_2016 & 97.5 & $\begin{array}{l}98.2 \\
92.0- \\
99.1\end{array}$ & 81.1-97.8 & 89.5-99.0 & $90.5-96.3$ & & $91.1-99.1$ & 79.4-98.3 & 88.1-98.6 & $88.0-96.7$ \\
\hline GX04/2017 & $\begin{array}{l}83.3- \\
88.0\end{array}$ & $\begin{array}{l}83.0- \\
87.9 \\
91.7- \\
94.8\end{array}$ & $\begin{array}{l}79.6-87.8 \\
81.1-89.4\end{array}$ & $\begin{array}{l}80.8-86.7 \\
90.5-94.3\end{array}$ & $\begin{array}{l}84.9-89.1 \\
91.3-94.2\end{array}$ & 78.1-97.2 & $\begin{array}{l}81.0-98.5 \\
90.7-99.1\end{array}$ & $\begin{array}{l}78.1-98.7 \\
80.0-98.9\end{array}$ & $\begin{array}{l}80.3-98.4 \\
91.0-99.5\end{array}$ & $\begin{array}{l}79.8-98.9 \\
88.8-98.3\end{array}$ \\
\hline GX01-2018 & $\begin{array}{l}84.1- \\
97.5\end{array}$ & $\begin{array}{l}83.6- \\
98.2 \\
92.1- \\
99.1\end{array}$ & $\begin{array}{l}79.8-97.2 \\
81.7-97.8\end{array}$ & $\begin{array}{l}82.5-98.4 \\
89.5-99.0\end{array}$ & $\begin{array}{l}83.5-96.8 \\
91.7-96.3\end{array}$ & $78.2-97.7$ & $\begin{array}{l}81.3-98.6 \\
91.1-99.3\end{array}$ & $\begin{array}{l}77.4-99.3 \\
80.0-99.4\end{array}$ & $\begin{array}{l}80.3-98.6 \\
88.1-99.5\end{array}$ & $\begin{array}{l}80.9-98.1 \\
89.2-97.9\end{array}$ \\
\hline GX02-2018 & $\begin{array}{l}84.2- \\
89.7\end{array}$ & $\begin{array}{l}83.6- \\
89.4 \\
92.3- \\
95.2\end{array}$ & $\begin{array}{l}80.7-90.4 \\
81.7-91.1\end{array}$ & $\begin{array}{l}82.4-88.6 \\
92.4-94.3\end{array}$ & $\begin{array}{l}86.4-91.6 \\
92.5-95.4\end{array}$ & 78.3-89.7 & $\begin{array}{l}81.4-89.7 \\
91.4-96.0\end{array}$ & $\begin{array}{l}78.0-91.7 \\
79.4-93.3\end{array}$ & $\begin{array}{l}80.6-89.4 \\
91.0-95.7\end{array}$ & $\begin{array}{l}82.3-92.0 \\
90.5-96.7\end{array}$ \\
\hline GX01-2019 & $\begin{array}{l}84.0- \\
90.9\end{array}$ & $\begin{array}{l}83.5- \\
90.7 \\
91.9- \\
95.4\end{array}$ & $\begin{array}{l}83.0-90.4 \\
83.9-91.1\end{array}$ & $\begin{array}{l}83.0-90.2 \\
90.0-93.8\end{array}$ & $\begin{array}{l}84.9-91.6 \\
92.9-95.4\end{array}$ & 78.3-97.6 & $\begin{array}{l}81.5-98.1 \\
91.1-99.2\end{array}$ & $\begin{array}{l}78.3-98.1 \\
80.6-97.2\end{array}$ & $\begin{array}{l}81.4-98.4 \\
90.0-100\end{array}$ & $\begin{array}{l}80.8-98.3 \\
90.9-98.8\end{array}$ \\
\hline GX02-2019 & $\begin{array}{l}83.3- \\
84.2\end{array}$ & $\begin{array}{l}83.0- \\
83.6 \\
91.7- \\
92.3\end{array}$ & $\begin{array}{l}79.6-83.0 \\
81.1-83.9\end{array}$ & $\begin{array}{l}80.8-83.0 \\
89.5-92.4\end{array}$ & $\begin{array}{l}83.4-86.4 \\
90.5-93.8\end{array}$ & 77.7-95.9 & $\begin{array}{l}80.8-96.9 \\
90.6-98.6\end{array}$ & $\begin{array}{l}78.1-97.8 \\
80.0-96.7\end{array}$ & $\begin{array}{l}80.8-96.5 \\
88.1-98.1\end{array}$ & $\begin{array}{l}80.6-97.8 \\
88.4-98.3\end{array}$ \\
\hline
\end{tabular}

Table 4 APPV reference strains used in this study 


\begin{tabular}{|c|c|c|c|}
\hline Strain & Accession number & Area & Collection date \\
\hline 2086 & MN099164 & Switzerland & 2006 \\
\hline NL1 & KX929062 & The Netherlands & 2012 \\
\hline 000515 & KR011347 & USA & 2014 \\
\hline ISDVDL2014016573 & KU194229 & USA & 2014 \\
\hline CN-CQ_11/39 & MF167292 & China & 2014 \\
\hline Bavaria_S5/9 & KU041639 & Germany & 2015 \\
\hline Ger-NRW_CT-59 & MF167290 & Germany & 2015 \\
\hline AUT-2016_C & KX778724 & Austria & 2016 \\
\hline APPV_GX-CH_2016 & KY652092 & China & 2016 \\
\hline KU16-2 & MF979135 & Korea & 2016 \\
\hline KU16-6 & MH509410 & Korea & 2016 \\
\hline GD1 & KX950761 & China & 2016 \\
\hline GD2 & KX950762 & China & 2016 \\
\hline GD3 & KY612413 & China & 2016 \\
\hline APPV-China/GZ01/2016 & KY475592 & China & 2016 \\
\hline APPV-China/GD-SD/2016 & KY475593 & China & 2016 \\
\hline APPV_GD & KY624591 & China & 2016 \\
\hline APPV-China/GD-GL/2016 & MH221022 & China & 2016 \\
\hline APPV-China/GD-HE/2016 & MH221023 & China & 2016 \\
\hline APPV-China/GD-HG/2016 & MH221024 & China & 2016 \\
\hline APPV-China/GD-SHM/2016 & MH221025 & China & 2016 \\
\hline APPV-China/GD-SHT/2016 & MH221026 & China & 2016 \\
\hline APPV-China/GD-ST/2016 & MH221027 & China & 2016 \\
\hline APPV_GER_01 & LT594521 & Germany & 2016 \\
\hline Ger-NRW_L277 & MF167291 & Germany & 2016 \\
\hline UNL.082017 & MK728876 & USA & 2017 \\
\hline 170711-1 & MN099169 & Switzerland & 2017 \\
\hline APPV_VIRES_NM01_C1 & MK378658 & China & 2017 \\
\hline HBtl1701 & MF377344 & China & 2017 \\
\hline GX04/2017 & MH102210 & China & 2017 \\
\hline APPV-China/SWU-XC/2017 & MH499645 & China & 2017 \\
\hline APPV-China/SWU-ZH/2017 & MH499647 & China & 2017 \\
\hline CH-GD2017 & MK629522 & China & 2017 \\
\hline GD-HJ-2017.04 & MK216752 & China & 2017 \\
\hline GD-LN-2017.04 & MK216753 & China & 2017 \\
\hline GD-YJHSEY2N & MK347475 & China & 2017 \\
\hline GD-ZW-2017.10 & MK216754 & China & 2017 \\
\hline YN01/2017 & MH378079 & China & 2017 \\
\hline JX-JM01-2018A01 & MG792803 & China & 2018 \\
\hline APPV-China/SWU-DY/2018 & MH499642 & China & 2018 \\
\hline APPV-China/SWU-KZ/2018 & MH499643 & China & 2018 \\
\hline APPV-China/SWU-MY/2018 & MH499644 & China & 2018 \\
\hline APPV-China/SWU-QL/2018 & MH499648 & China & 2018 \\
\hline APPV-China/SWU-YB/2018 & MH499646 & China & 2018 \\
\hline GD-BH02-2018 & MH520668 & China & 2018 \\
\hline GD-BZ01-2018 & MH493896 & China & 2018 \\
\hline GD-LDCT1 & MK347474 & China & 2018 \\
\hline GD-CT4 & MN584737 & China & 2018 \\
\hline GD-DH01-2018 & MH493895 & China & 2018 \\
\hline GD-MH01-2018 & MH493894 & China & 2018 \\
\hline 180416 & MN099170 & Switzerland & 2018 \\
\hline AH-GL-2018.01 & MK216750 & China & 2018 \\
\hline AH-SG-2018.01 & MK216751 & China & 2018 \\
\hline HN2018 & MH885413 & China & 2018 \\
\hline GX01-2018 & MH715893 & China & 2018 \\
\hline GX02-2018 & MK453045 & China & 2018 \\
\hline GX01-2019 & MN564752 & China & 2019 \\
\hline GX02-2019 & MN729215 & China & 2019 \\
\hline
\end{tabular}

Table 5 Recombination analysis of APPV complete genomes 


\begin{tabular}{llll}
\hline Strain & Major Parent/Similarity(\%) & Minor Parent/Similarity $(\%)$ & Breakpoint position in alignment \\
\hline GD-BZ01-2018 & AH-SG-2018/98.8 & GD-CT4/100 & $2138-2537 / 3253-3678$ \\
& GD-MH01-2018/98.6 & APPV_VIRES_NM01_C1/97.9 & $4712-4782 / 6590-6618$ \\
& GD-CT4/95.7 & GD2/93.4 & $9950-9984 / 11400-361$ \\
GD-DH01-2018 & GD-CT4/99.4 & GD2/99.4 & $9834-9868$ \\
YN01/2017 & KU16-6/97.2 & APPV-China/GD-SD/2016/97.2 & $6453-6498$ \\
JX-JM01-2018A01 & HBtl1701/97.3 & APPV_GX-CH_2016/95.8 & $10895-11004 / 220-570$ \\
& HBtl1701/95 & CN-CQ_11/39/97.1 & $845-1113 / 2240-2325$ \\
& GX01-2018/93.7 & APPV_VIRES_NM01_C1/97.3 & $3127-3294 / 3809-3870$ \\
& HBtl1701/96.2 & CN-CQ_11/39/97.7 & $4196-4618 / 57848-5937$ \\
GD-LN-2017.04 & GX04/2017/93.5 & GX01-2018/98.6 & $6236-6342 / 9095-9198$ \\
GD2 & APPV-China/GD-SHM/2016/99.7 & GD-HJ-2017.04/99.9 & $9352-11053 / 2118-2771$ \\
\hline
\end{tabular}

Figures

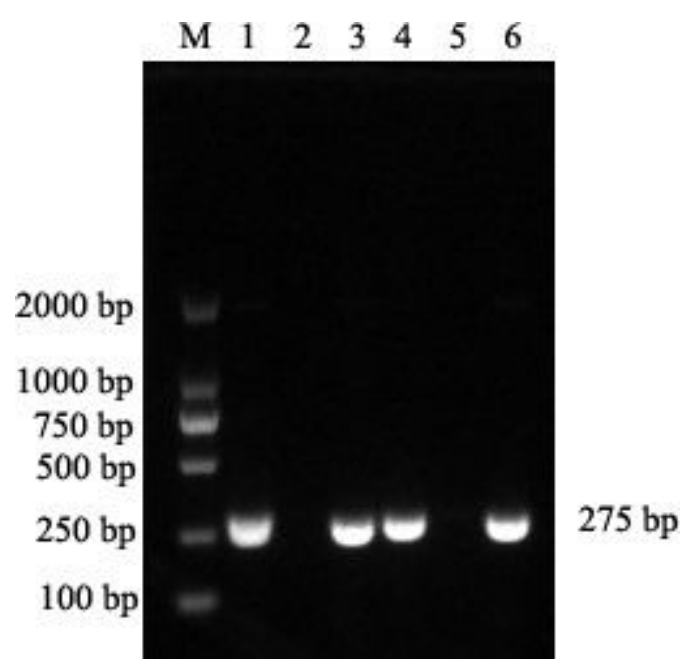

\section{Figure 1}

Detection results of clinical samples for APPV by RT-PCR M: DL 2000 DNA Marker; 1: Positive control; 2: Negative control; 3-6: Clinical samples

$\begin{array}{lllllllllllll}\text { M } & 1 & 2 & 3 & 4 & 5 & 6 & 7 & 8 & 9 & 10 & 11\end{array}$

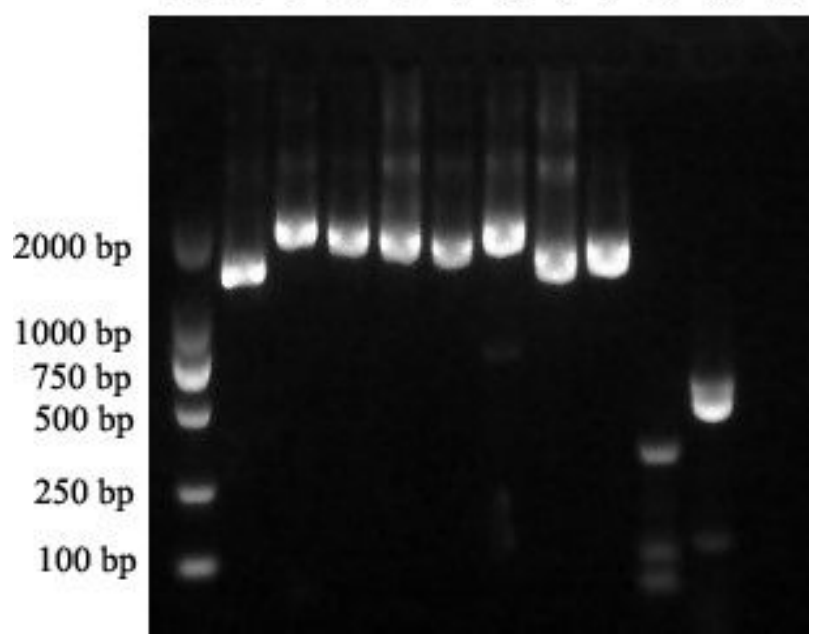

\section{Figure 2}

Amplification of APPV complete genome by RT-PCR M: DL 2000 DNA Marker; 1-8: Fragments of ORF; 9: 5' UTR; 10: 3' UTR; 11: Negative control 

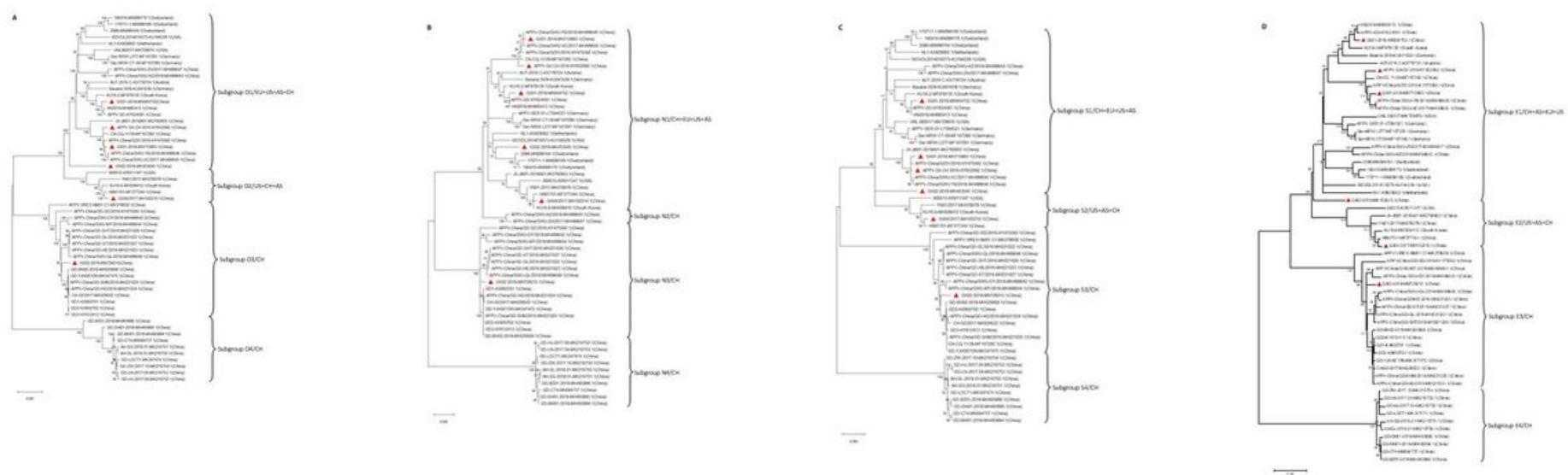

Figure 3

Phylogenetic trees based on the complete genome (A), Npro (B), Erns (C) and E2 (D) gene sequences of APPV strains from Guangxi province and other reference strains available in GenBank. Bootstrap values are shown at the nodes. Red solid triangle represents APPV strain from Guangxi province, China. AS: Asia; CH: China; EU: Europe; US: USA

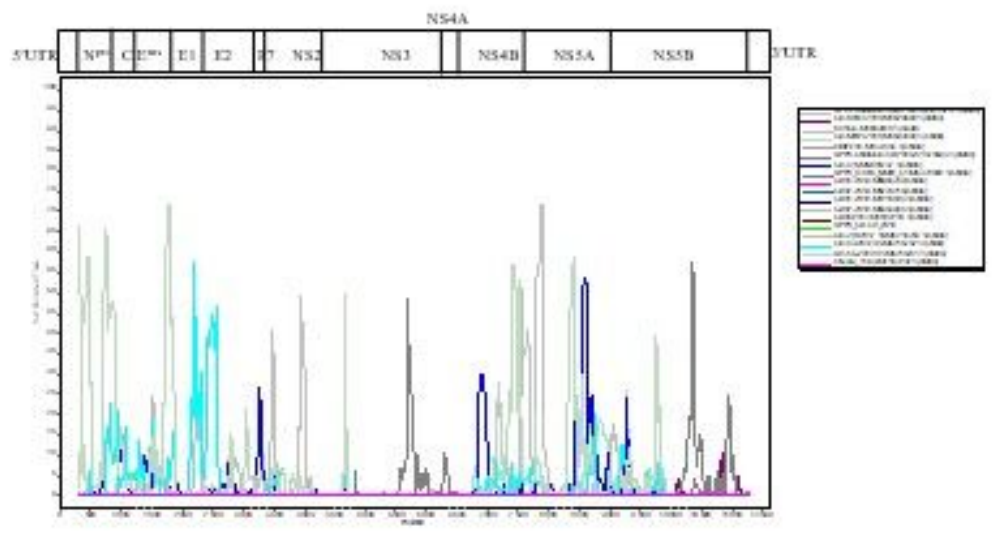

\section{Figure 4}

Recombination analyses on the complete genomes of six APPV strains from Guangxi province. Potential recombination events were identified using Recombination Detection Program 4 (RDP4) and then examined using similarity plots and bootstrap analyses Simplot 3.5.1. The major and minor parents were represented in table 5 